\title{
Mortality benefits and overdiagnosis estimates for women attending breast screening
}

\author{
N Ormiston-Smith ${ }^{* 1,3}, \mathrm{H} \mathrm{Scowcroft}^{2}$ and C S Thomson ${ }^{1}$ \\ ${ }^{1}$ Cancer Research UK, Statistical Information Team, The Angel Building, 407 St John Street, London EC1V 4AD, UK and ${ }^{2}$ Cancer \\ Research UK Communications Department, London, UK
}

Sir,

The recent well-publicised Marmot review (Independent UK Panel on Breast Cancer Screening, 2012; Marmot et al, 2013) provides much-needed insight into the estimated harms and benefits of breast screening but, as it is an intention-to-treat analysis, sets out its main findings in terms of women invited to screening. Such an analysis does not need to adjust for either non-compliance or loss to follow-up, and is useful for assessing the effect of the intervention on society, but it is not designed to portray the likely impact of screening on an individual.

In practice, women do not accrue benefits or harms of breast screening merely by receiving a screening invitation-these occur only during or after mammography itself. Thus, here we present an alternative set of statistics (derived from those within the Marmot review) estimating the risks and benefits to women of attending breast screening. It is clear in the Marmot review that the figures are subject to a large amount of uncertainty, which also applies to the figures presented here.

The absolute reduction in risk of dying from breast cancer for women attending screening can be estimated by adjusting the absolute risk reduction in those invited to screening $(0.43 \%)$ for the coverage (coverage rate in the NHSBSP is $77 \%$ for England in 2010-2011 (The Health and Social Care Information Centre, Screening and Immunisations Team, 2012)). Thus, as presented in the Marmot review, the estimated breast cancer mortality reduction is $0.56 \%$ ( $0.43 \%$ divided by $0.77 \%)$, or 56 breast cancer deaths prevented per 10000 women attending screening.

We have applied a similar calculation to the estimated percentage of women with an overdiagnosis (1.29\%) based on the intention-to-treat analysis. This gives an estimate of $1.68 \%$ ( $1.29 \%$ divided by $0.77 \%$ ) overdiagnosis, or 168 per 10000 women attending screening.
However, the same calculation cannot simply be repeated to estimate the number of breast cancers and DCIS diagnosed in women attending breast screening in the United Kingdom, as socioeconomic deprivation is associated with both the risk of breast cancer and the likely attendance at screening (both are higher in more affluent populations) (Maheswaran et al, 2006; National Cancer Intelligence Network, 2008).

We have therefore assumed that the risk of breast cancer in women who attend breast screening is $10 \%$ higher than generally for the whole population of women invited for screening. We feel this is reasonable, as a large range of estimates of the increased risk in attenders versus non-attenders has been reported-this figure is roughly in the middle of these estimates (Collette et al, 1984; van Dijck et al, 1996; Puliti et al, 2008). Thus, we estimate there will be 749 breast cancers diagnosed in 10000 women attending screening (681 breast cancers per 10000 women in those invited for screening from the Marmot review $+10 \%)$.

To demonstrate these harms and benefits we have compared what would happen to 10000 women attending screening in the United Kingdom after 20 years with what would happen had they not been able to attend if there were no screening programme available to them (numbers are rounded to whole numbers).

- Seven hundred and forty-nine women will be diagnosed with breast cancer and will receive treatment.

Of these 749 women,

- One hundred and fifty-seven will die from breast cancer, 56 fewer than in the group not attending screening.

*Correspondence: N Ormiston-Smith, E-mail: nick.ormiston-smith@cancer.org.uk

${ }^{3}$ Member of the Secretariat for the Independent UK Panel on Breast Cancer Screening 
Of the 592 who survive,

- Fifty-six patients will have their life extended by screening, see calculation above.

- One hundred and sixty-eight will be diagnosed and treated for a cancer that would not have caused problems in their lifetime ('overdiagnosed'), see calculation above.

- Three hundred and sixty-nine, the remainder, will be diagnosed with and treated for a cancer that would have been picked up later without screening.

If the same 10000 women were not able to attend screening, then after 20 years

- Five hundred and eighty-two will be diagnosed with breast cancer, and will receive treatment, that is, the 749 minus the number of overdiagnosed cases.

- One hundred and sixty-eight women will have a breast cancer they never know about and that will not cause any harm during their lifetime.

Of the 582 women diagnosed,

- Two hundred and thirteen women will die from breast cancer, 56 more than in the group attending screening.

- Three hundred and sixty-nine, the remainder, will be treated for cancer and will survive.

These figures give further insight into the benefits and harms of breast screening, but from the individual point of view. We hope they will inform the millions of women invited for screening in the United Kingdom each year, and those around the world, and help to make their decision about whether or not to go for screening. Further information can be found on Cancer Research UK's website (Cancer Research UK, 2012).

\section{REFERENCES}

Cancer Research UK (2012) Breast Screening Review. Available at www.cruk.org/breastscreeningreview (accessed May 2013).

Collette HJA, Rombach JJ, Day NE, de Waard F (1984) Evaluation of screening for breast cancer in a non-randomised study (The DOM Project) by means of a case-control study. Lancet 1(8388): 1224-1226.

Independent UK Panel on Breast Cancer Screening (2012) The benefits and harms of breast cancer screening: an independent review. Lancet 380(9855): 1778-1786.

Maheswaran R, Pearson T, Jordan H, Black D (2006) Socioeconomic deprivation, travel distance, location of service, and uptake of breast cancer screening in North Derbyshire, UK. J Epidemiol Commun Health 60(3): 208-212.

Marmot G, Altman DG, Cameron DA, Dewar JA, Thompson SG, Wilcox M. The Independent UK Panel on Breast Cancer Screening (2013) The benefits and harms of breast cancer screening: an independent review. $\mathrm{Br} J$ Cancer 108(11): 2205-2240.

National Cancer Intelligence Network (2008) Cancer Incidence by Deprivation England, 1995-2004. Available at http://www.ncin.org.uk/ cancer_type_and_topic_specific_work/topic_specific_work/equality.

Puliti D, Miccinesi G, Collina N, De Lisi V, Federico M, Ferretti S, Finarelli AC, Foca F, Mangone L, Naldoni C, Petrella M, Ponti A, Segnan N, Sigona A, Zarcone M, Zorzi M, Zappa M, Paci E (2008) Effectiveness of service screening: a case-control study to assess breast cancer mortality reduction. Br J Cancer 99(3): 423-427.

The Health and Social Care Information Centre, Screening and Immunisations Team (2012) The NHS Information Centre. Available at http://www.hscic.gov.uk/searchcatalogue?productid=4899\&q=breast + screening + england $+2010-2011$ \&sort $=$ Relevance\&size $=10$ \&page $=1 \#$ top .

van Dijck JA, Verbeek AL, Beex LV, Hendriks JH, Holland R, Mravunac M, Straatman H, Werre JM (1996) Mammographic screening after the age of 65 years: evidence for a reduction in breast cancer mortality. Int $J$ Cancer 66(6): 727-731.

(c) (1) (2) (2) This work is licensed under the Creative Commons Attribution-NonCommercial-Share Alike 3.0 Unported License. To view a copy of this license, visit http://creativecommons. org/licenses/by-nc-sa/3.0/ 